\title{
Contralateral complete L5 palsy following ipsilateral L4 selective transforaminal epidural block
}

\author{
Young-Jin Yi, Sang-Soo Kang, Young-Joon Yoon, and Keun-Man Shin \\ Department of Anesthesiology and Pain Medicine, Kangdong Sacred Heart Hospital, Hallym University College of Medicine, Seoul, \\ Korea
}

The exact origins of pain and neurologic findings in patients with herniated discs are not completely understood. Mechanical compression and inflammatory processes are the most well known possible causes of radicular pain. Generally, clinical symptoms correspond to the findings on radiologic studies. But in rare cases, clinical findings do not match radiological findings. We report a rare case of contralateral complete L5 palsy following ipsilateral L4 selective transforaminal epidural block in a patient with central protrusion of the L3-4 intervertebral disc and spinal stenosis with combined disc extrusion to the left lateral recess and lower migration. The exact mechanism remains unclear, and the suspected mechanism is compressive force that could have been aggravated via epidural injection. Here, we report an unexpected result of an epidural block and review the relevant literatures.

A 70-year-old man presented with a 3-month history of lower back pain and radicular pain on the posterolateral aspect of the leg bilaterally. The leg pain was dominant on the right side. The patient also occasionally experienced mild bilateral buttock and posterior thigh pain. The symptoms were aggravated by prolonged standing and walking and relieved by rest. Physical examination revealed normal reflexes and strength. The patient had no lower extremity weakness. The straight leg raising test and Lasegue maneuver were negative bilaterally. Other findings on neurological examination were normal except for hypoesthesia on the medial side of the right leg. Lumbar spine magnetic resonance imaging (MRI) revealed mild disc bulging at the L12, L2-3, L4-5, and L5-S1 intervertebral disc levels, central pro- trusion at the L3-4 intervertebral disc with combined disc extrusion to the left lateral recess and lower migration, and spinal stenosis due to disc protrusion, bulging and facet hypertrophy at the L3-4 level (Fig. 1). Although the patient's predominant symptoms did not correlate with the MRI findings, we planned a left L4 selective transforaminal epidural block based on the MRI findings. To begin the procedure, a $100 \mathrm{~mm}, 20-\mathrm{G}$ Tuohy needle was advanced toward the left $\mathrm{L} 4$ intervertebral foramen. The spreading of contrast medium (Omnipaque 300) $4 \mathrm{ml}$ was observed with real-time fluoroscopy. No arterial or venous uptake and no subarachnoid spread of contrast medium were observed. A mixture of normal saline $4 \mathrm{ml}$ with $2 \%$ mepivacaine $0.5 \mathrm{ml}$ was injected for irrigation, followed by an injection of a mixture of normal saline $4 \mathrm{ml}$ and $20 \mathrm{mg}$ of triamcinolone $1 \mathrm{ml}$. There was no immediate numbness or weakness in the lower extremities, and the patient's symptoms were relieved after the procedure. Two hours later, however, the patient complained of weakness of the right leg. Physical examination revealed hypoesthesia in the L5 dermatome of the right leg and complete motor weakness of dorsiflexion of the right ankle. MRI was immediately repeated at the L3-4 and L4-5 levels, but no changes or abnormalities were observed compared to the previous study. Emergency decompression was scheduled. Bilateral partial L3 laminectomy with left L3-4 discectomy was performed, and disc material was successfully removed by the neurosurgeon. In the day following the surgery, the patient experienced a slow but steady neurological recovery. A week later, his neurological deficit was recovered and the patient was discharged from the

Corresponding author: Keun-Man Shin, M.D., Ph.D., Department of Anesthesiology and Pain Medicine, Kangdong Sacred Heart Hospital, Hallym University Medical Center, 445, Gil-dong, Gangdong-gu, Seoul 134-701, Korea. Tel: 82-2-2224-2659, Fax: 82-2-474-0956, E-mail: paintx@ naver.com

(c) This is an open-access article distributed under the terms of the Creative Commons Attribution Non-Commercial License (http:// creativecommons.org/licenses/by-nc/3.0/), which permits unrestricted non-commercial use, distribution, and reproduction in any medium, provided the original work is properly cited. 

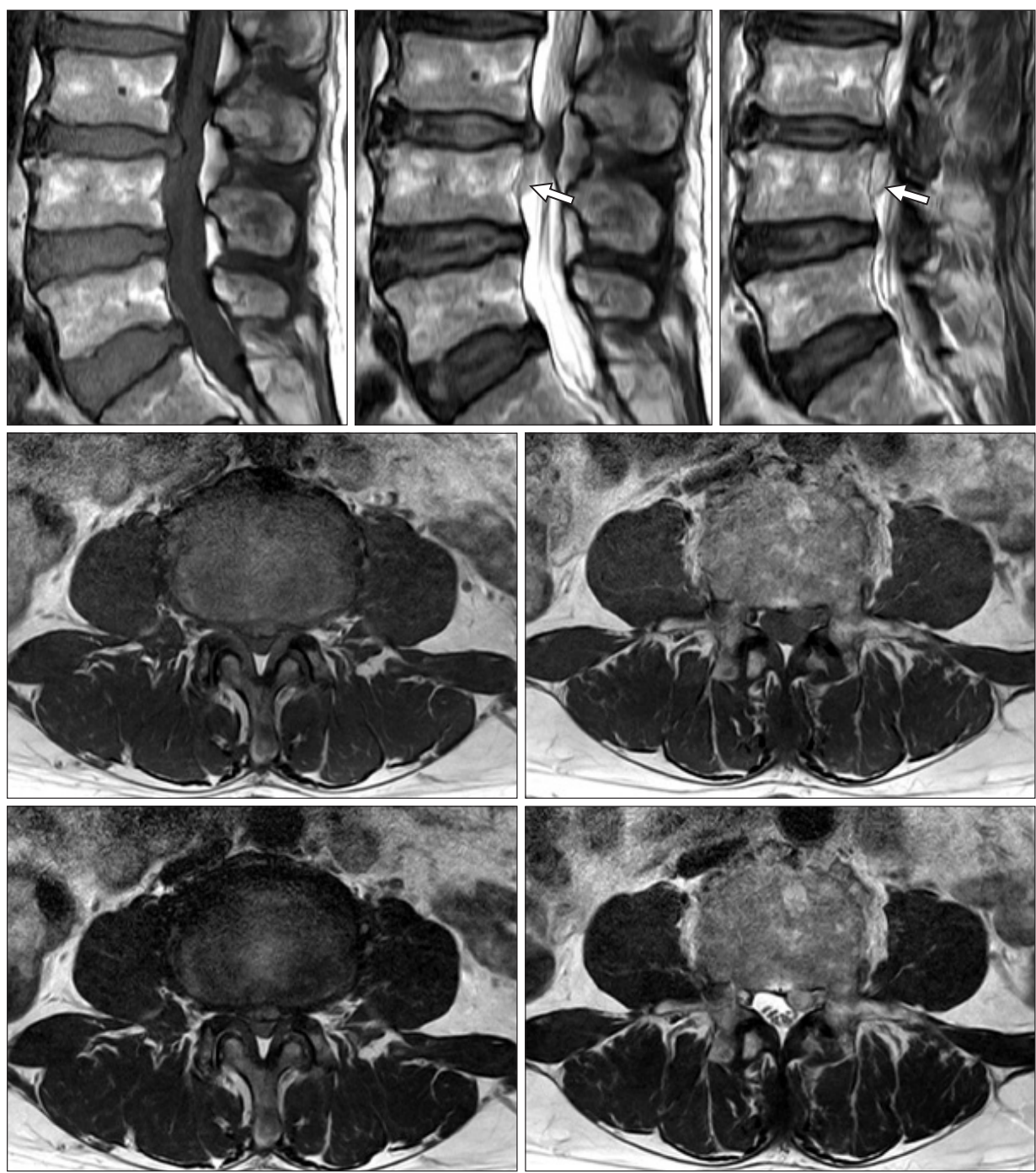

Fig. 1. Preoperative lumbar magnetic resonance imaging (MRI) reveals spinal stenosis due to central disc protrusion, bulging and facet hypertrophy at the L3-4 level, and combined disc extrusion to the left lateral recess and lower migration (White arrows indicate extruded and migrated disc material). Additional findings on the MRI are mild disc bulging at the L1-2, L2-3, L4-5, and L5-S1 intervertebral disc levels. hospital. He resumed normal activity one month later.

There are a few existing reports describing contralateral symptoms from lumbar disc herniation. Choudhury et al. [1] explained that spondylotic changes and stenosis contralateral to the side of disc herniation caused displacement and impaction of the dural sac with the emerging nerve roots in a narrowed lateral recess. Kornberg [2] proposed that the herniated disc shifts the theca to the contralateral side, forming a wedge-like shape, and pressure applied on the nerve root as a result of contact force leads to radicular pain. Sucu and Gelal [3] hypothesized that contralateral findings may be related to traction forces rather than contact forces. A common finding of previously proposed mechanisms such as spondylotic changes and stenosis, contract force, and traction force is a compressive effect. Nakagawa et al. [4] proposed inflammatory theory rather than compressive theory. In two cases of operative findings, they reported that the nerve root of the symptomatic side, contralateral to the side of the disc herniation, had been compressed to the superior facet by the herniated disc from the opposite side, with resultant inflammatory findings, such as fibrosis, adhesion, redness, and swelling. In the present case, as the patient's neurological deficit recovered slowly after ipsilateral discectomy and removal of migrated disc materials, the authors think that the mechanism is more likely to be explained by compressive theory than by inflammatory theory.

A few reports exist of paraplegia following lumbar interlaminar or transforaminal epidural injection. Most authors proposed that the mechanism of this complication is ischemia of the terminal cone due to accidental suppression of the medullary blood supply. However, we examined an MRI immediately after the onset of palsy and observed no evidence of spinal cord ischemia. Acute transient monoplegia after lysis of epidural adhesions using a Racz catheter with failed back surgery syndrome has been reported [5]. Motor and sensory function in the right 
lower limb returned after 5 days. It was suspected that large volumes of fluid injected during neuroplasty could have caused transient nerve injury from compression within loculated epidural compartments. The critical pressure level for compressioninduced impairment has still not been determined. The spinal nerve roots may be even more susceptible to compression than peripheral nerves because they are located in a closed space. In the vertebral column, this means that the canal should be narrowed or the contents increased significantly, as in the case of disc herniation. Although loculation of the contrast-containing injected fluid was not observed during the fluroscopically guided epidural block and the surgery, our thinking is that injected fluid materials may have caused some degree of compressive effect within the narrowed spinal canal in the case discussed here.

This is a rare case report of contralateral complete L5 palsy following ipsilateral L4 selective transforaminal epidural block. When intervention is planned as in this situation, providers should consider the MRI findings carefully, pay close attention to any changes in the patient's symptoms, and monitor the situation closely for possible complications.

\section{References}

1. Choudhury AR, Taylor JC, Worthington BS, Whitaker R. Lumbar radiculopathy contralateral to upper lumbar disc herniation: report of 3 cases. Br J Surg 1978; 65: 842-4.

2. Kornberg M. Sciatica contralateral to lumbar disk herniation. Orthopedics 1994; 17: 362-4.

3. Sucu HK, Gelal F. Lumbar disk herniation with contralateral symptoms. Eur Spine J 2006; 15: 570-4.

4. Nakagawa Y, Yoshida M, Kawakami M, Ando M, Hashizume H, Minamide A, et al. Posterior endoscopic surgery for lumbar disc herniation with contralateral symptoms - a report of two cases. Minim Invasive Neurosurg 2006; 49: 282-5.

5. Mirovsky Y, Halperin N. Eccentric compression of the spinal canal causing dominantly contralateral-side symptoms. J Spinal Disord 2000; 13: 174-7. 